\title{
AJCC v7 Stage
}

National Cancer Institute

\section{Source}

National Cancer Institute. A/CC v7 Stage. NCI Thesaurus. Code C90530.

A cancer stage defined according to the AJCC 7th edition criteria. 